\title{
FACTS devices in Distributed Generation
}

\author{
${ }^{1}$ Melchor Gómez, ${ }^{2}$ Oihane Abarrategui, ${ }^{3}$ Inmaculada Zamora \\ Department of Electrical Engineering \\ University of the Basque Country (UPV/EHU) \\ ${ }^{1,2}$ E.U.I.T.M. y O.P de Barakaldo. c/ Colina de Beurko, s/n (48902 Barakaldo) \\ ${ }^{3}$ E.T.S.I. de Bilbao. c/ Alda. Urquijo s/n 48013 Bilbao \\ 1 phone:+34 946014970, fax:+34 946017800, e-mail: iepgopem@lg.ehu.es \\ 2phone:+34 946014971, fax:+34 946017800, e-mail: gauoian@yahoo.es \\ 3phone:+ 34 946014063, fax:+34 946014200, e-mail: iepzabei@lg.ehu.es \\ Spain
}

\begin{abstract}
The FACTS devices control the interrelated parameters that rule the operation of the transmission systems, including the serial impedance, the derivation impedance, the current, the tension, the phase angle and the muffling of oscillations to different frequencies under the nominal frequency. The FACTS technology has a collection of controllers, that can be used individually or co-ordinated with other controls installed in the network, thus permitting to profit better of the network's characteristics of control.

The configurations of compensators based on switched inverters, called static compensators of reactive power, are today the most used in the electric current transmission systems. The apparition of high-powered electronic converters, makes possible the generation or absorption of reactive power without using banks of condensers or inductors.

The STATCOM is a device connected in derivation that can operate in networks of transport as well as of distribution. The functions will depend on the type of network in which it will be installed, it could fulfill functions of reactive power control and tension regulation, or other functions as power factor regulator, shock absorber of system oscillations or filtering of harmonics, depending on the systems applications and the tension level we wish to install.
\end{abstract}

\section{Key words}

FACTS, Energy Quality, STATCOM, CUSTOM POWER, Active Filter.

\section{Introduction}

The solutions to improve the quality of supply in the electrical networks with distributed generation go through the application of the developments in semiconductor power devices, that is to say, the utilization of static power converters in electrical energy networks. The technological advances in power semiconductors are permitting the development of devices that react more like an ideal switch, totally controllable, admitting high frequencies of commutation to major levels of tension and power.

On the other hand, large advances in auxiliary technologies devices of digital control, DSP's ( Digital Signal Processor ), circuits of programmable logic and in techniques of advanced control. These technological developments, united to a tendency of reduction of cost of the power semiconductors, are permitting to undertake a new practical way to practice topologies of conversion of capable energy to give competitive solutions to the related problems, with the interconnection of new energy sources in the networks of high and medium tension and with improved the quality of the electricity supply.

The concept of distributed Generation is generally associated to the development of the renewable energy sources and other alternative sources as the fuel piles, is another factor to keep in mind in the development and configuration of the electrical system, that will need an important electronic equipment based on power converters that facilitate the integration of these sources of energy, without damaging over the reception quality of the users connected to the electricity network.

The use of static power converters in electricity networks has the potential of increasing the capacity of transmission of the electric lines and improving the supply quality of the electric energy. The devices used to achieve this, are the FACTS (Flexible Alternating Current Transmission Systems). 
According to the IEEE the definition of these FACTS devices is the following:"Alternating Current Transmission Systems static and other power electronics controllers to improve the control and increase the capacity of power transfer. "

The FACTS controllers offer great opportunities to regulate the transmission of alternating current (AC), increasing or diminishing the power flow in specific lines and responding almost instantaneously to the stability problems. The potential of this technology is based on the possibility of controlling the route of the power flow and the ability of connecting networks that are not adequately interconnected, giving the possibility of trading energy between distant agents.

\section{FACTS in Active Distribution Systems}

In the new market of Active Distribution, the flexibility of the transmission depending on the prices of the electric energy in each moment is imposed. The fact that the energy can vary dynamically in the way from generation to consumption requires a bigger margin of the lines design, or at least, a major control of the energy they transmit.

Narrowly related to the FACTS term is that of CUSTOM POWER, controllers based on solid state technologies that are designed to operate in medium and low tension levels, whose main objective is to improve the quality of service in distribution networks. The advances in controllable semiconductors, microcontrollers, signal processors and energy storage technologies have permitted the design of new devices that present a faster dynamic answer and a more accurate approach in basic and important functions such as the tension regulation, the reactive power compensation, the harmonics distortion rate reduction or the limitation of short circuit currents.

The solutions to improve the energy quality (PQ- Power Quality) at the load side is of great important when the production processes get more complicated and require a bigger liability level, which includes aims like to provide energy without interruption, without harmonic distortion and with tension regulation between very narrow margins. The devices that can fulfil these requirements are the Custom Power; a concept that we could include among the FACTS, but that is different to them because of their final use. In fact the topologies that they employ are identical to the ones in the FACTS devices with little modifications and adaptations to tension levels, therefore they are most oriented to be used in distribution networks of low and medium tension, sometimes replacing the active filters.

\section{A. ADVANTAGES AND OPERATIVITY OF FACTS DEVICES}

The following features resume the main advantages of the FACTS devices:
- They allow a greater control over the power flow, routing it through predetermined routes.

- It is possible to operate at safe load levels (without overload) near to the thermical limits of the transmission lines.

- Bigger capacity of power transmission between controlled areas, thus reducing considerable the reserve margin.

- They increase the system security by increasing the transitory stability limit.

- They dampf the system oscillations that harm the equipment and limit the available capacity.

- They answer phase to the network condition changes and control the power flow in real time.

- They provide the network of flexibility to install new generating plants.

- They provide security in the connections through the joining lines between enterprises and neighbour areas.

- Great flexibility in the three operative status of the system: pre-fault, fault, post-fault and capacity to control transitory status and to impact phase in post fault status.

The FACTS devices, as any other component of the system, introduce modes of oscillation in the behaviour, and becomes more complicated to operate; this can lead to unwanted interactions between equipment. Due to it, the coordination of all the controllers in the system including the FACTS devices must to be foreseen. The inclusion of this type of elements in the power system offers some advantages but also brings complexities that must be taken away for the safe operation of the system.

There are three factors to be considered before installing a FACTS device:

- The type of device

- The capacity required

- The location that optimize the functioning of the device

Of these factors, the last one is of great importance, because the desired effect and the proper features of the system depend on the location of FACTS.

\section{B. CLASSIFICATION}

There are different classifications for the FACTS devices: 
- Depending on the type of connection to the network. The FACTS device can differentiate four categories;

-Serial Controllers.

-Derivation Controllers

-Serial to Serial Controllers

-Serial-derivation Controllers
-Second generation: Semiconductors with ignition and extinction controlled by door (GTO's, MCTS, IGBTS, IGCTS, etc. ).

These two classifications are independent, existing for example, devices of a group of the first classification that can belong to various groups of the second classification. In the table 1 . you can see the summary of the main devices.

- Depending on technological features, the FACTS devices can be divided into two generations:

-First generation: Uses thyristors with ignition controlled by door ( SCR)

\begin{tabular}{|c|c|}
\hline FACTS Devices & Attributes of Control \\
\hline $\begin{array}{l}\text { Static compensator of VAR's (SVC) (TCR, } \\
\text { TCS, TRS) }\end{array}$ & $\begin{array}{l}\text { Voltage control and stability, compensation of VAR's, muffling of } \\
\text { oscillations }\end{array}$ \\
\hline $\begin{array}{l}\text { Thyristor Controlled Series Compensation } \\
\text { (TCSC, TSSC) }\end{array}$ & $\begin{array}{l}\text { Current Control, muffling of oscillations, transitory, dynamics and of } \\
\text { tension stability, limitation of fault Current }\end{array}$ \\
\hline $\begin{array}{l}\text { Thyristor Controlled Reactor Series (TCSR, } \\
\text { TSSR) }\end{array}$ & $\begin{array}{l}\text { Current Control, muffling of oscillations, transitory, dynamics and of } \\
\text { Voltage stability, limitation of fault Current }\end{array}$ \\
\hline $\begin{array}{l}\text { Thyristor Controlled Phase Shifting } \\
\text { Transformer (TCPST o TCPR) }\end{array}$ & $\begin{array}{l}\text { Control of active power, muffling of oscillations, transitory, dynamics } \\
\text { and of Voltage stability }\end{array}$ \\
\hline $\begin{array}{l}\text { Thyristor Controlled Voltage Regulator } \\
\text { (TCVR) }\end{array}$ & $\begin{array}{l}\text { Control of reactive power, voltage control, muffling of oscillations, } \\
\text { transitory, dynamics and of Voltage stability }\end{array}$ \\
\hline $\begin{array}{l}\text { Thyristor Controlled Voltage Limited } \\
\text { (TCVL) }\end{array}$ & Limit of transitory and dynamic voltage \\
\hline $\begin{array}{l}\text { Synchronous Static } \quad \text { Compensator } \\
\text { (STATCOM Without storage) }\end{array}$ & $\begin{array}{l}\text { Voltage control , compensation of VAR's, muffling of oscillations, } \\
\text { stability of voltage }\end{array}$ \\
\hline $\begin{array}{l}\text { Synchronous } \quad \text { Static } \\
\text { (STATCOM With storage) }\end{array}$ & $\begin{array}{l}\text { Voltage control and stability, compensation of VAR's, muffling of } \\
\text { oscillations, transitory, dynamics and of tension stability }\end{array}$ \\
\hline $\begin{array}{l}\text { Static Synchronous Series Compensator } \\
\text { (SSSC Without storage ) }\end{array}$ & $\begin{array}{l}\text { Current Control, muffling of oscillations, transitory, dynamics and of } \\
\text { Voltage stability, limitation of fault Current }\end{array}$ \\
\hline $\begin{array}{l}\text { Static Synchronous Series Compensator } \\
\text { (SSSC With storage) }\end{array}$ & $\begin{array}{l}\text { Current Control, muffled of oscillations, transitory, dynamics and of } \\
\text { tension stability }\end{array}$ \\
\hline Unified Power Flow Controller (UPFC) & $\begin{array}{l}\text { Control of active and reactive power, voltage control, compensation of } \\
\text { VAR's, muffling of oscillations, transitory, dynamics and of voltage } \\
\text { stability, limitation of fault Current }\end{array}$ \\
\hline $\begin{array}{l}\text { Interline Power Flow Controller (IPFC) or } \\
\text { Back to Back (BtB) }\end{array}$ & $\begin{array}{l}\text { Control of reactive power, voltage control, muffling of oscillations, } \\
\text { transitory, dynamics and of voltage stability }\end{array}$ \\
\hline Tobl 1 & Second generation \\
\hline
\end{tabular}

\section{TYPES OF NETWORK CONNECTION}

Serial Controllers: Can consist of a variable impedance as a condenser, coil, etc. or a variable electronics based source at a fundamental frequency. The principle of operation of all the serial controllers is to inject a serial tension to the line. A variable impedance multiplied by the current that flows through it represents the serial tension. While the tension is in cuadrature with the line current the serial controller only consumes reactive power; any other phase angle represents management of active power. A typical controller is Serial Synchronous Static Compensator (SSSC). 


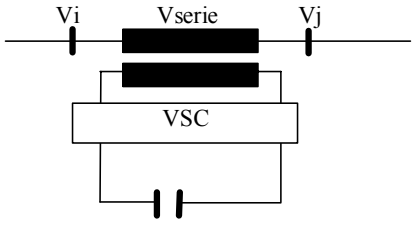

Fig. 1. Serial Controllers

Controllers in derivation. As it happens with the serial controller, the controller in derivation can consist of a variable impedance variable, variable source or a combination of both. The operation principle of all controllers in derivation is to inject current to the system in the point of connection. A variable impedance connected to the line tension line causes variable current flow, representing an injection of current to the line. While the injected current is in cuadrature with the line tension, the controller in derivation only consumes reactive power; any other phase angle represents management of active power. A typical controller is Synchronous Static Compensator (STATCOM)

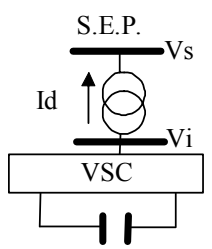

Fig. 2. Controllers in derivation.

Serial-serial Controllers. This type of controller can be a combination of co-ordinated serial controllers in a multiline transmission system, or can also be am unified controller in which the serial controllers provide serial reactive compensation for each line also transferring active power between lines through the link of power. The active power transmission capacity that presents a unified serial-serial controller or line feed power controller (Also called BtB-Black to Black), makes possible the active and reactive power flow balance and makes the use of transmission bigger. In this case the term "unified" means that the DC terminals of the converters of all the controllers are connected to achieve a transfer of active power between each other. A typical controller is the Interline Power Flow Compensator (IPFC)

Serial-derivation Controllers: This device can be a combination of serial and derivation controllers separated, co-ordinately controlled or a unified power flow controller with serial and derivation elements. The principle of operation of the serial-derivation controllers is to inject current to the system through the component in derivation of the controller, and a serial tension with the line utilizing the serial component. When the serial and derivation controllers are unified, they can have an exchange of active power between them through their link. A typical controller is Unified Power Flow Controller (UPFC) which incorporating functions of filtering and conditioning, becomes a Universal Power Lines Conditioner (UPLC).

\section{Second Generation FACTS devices}

The first generation FACTS devices work like passive elements using impedances or tap changer transformers controlled by thyristors. The second generation FACTS devices work like angle and module controlled voltage sources and without inertia, based in converters, employing electronic tension sources (three-phase inverters, auto-switched voltage sources, (SVS) Synchronous Voltage sources or (VSC) Voltage Source Control) fast proportioned and controllable and static synchronous voltage and current sources.

The main difference between first and second generation devices is the capacity to generate reactive power and to interchange active power. In the first generation, these abilities are exclusive for SVC and TCSC, are compensators of reactive but are not capable exchanging active power with the system, or in the case of the TCSP, the can exchange active or reactive power, but are not capable generating reactive power. The second generation has the inherent capacity, as a synchronous machine, to exchange active and reactive power and with the system, furthermore, to generate or absorb it automatically, having as consequence reactive compensation without condensers or alternating current coils. The real power has to be exchanged through the AC system.

Simplifying, the difference between these generations of FACTS devices is the flexibility and the response dynamic. The second generation of FACTS devices adapt to improve to changes in the topology or in the point of work of the network and the response dynamics is faster. Nevertheless, the second devices of this generation of FACTS are more complex and expensive.

The following controllers belong to this second generation:

\section{-STATCOM ( Static Synchronous Compensator ) \\ -SSSC (Static Synchronous Serious Compensator ) \\ -UPFC (Unified Power Flow Controller ).}

The SSSC is identical to the STATCOM ( in equipment and operation ) but employs them in a different disposition (serial) and UPFC has two STATCOM one serial and the other in derivation.

\section{A. STATCOM(Static Synchronous Compensator )}

It is a device connected in derivation, basically composed of a coupling transformer, that serves of link between the electric Power system (SEP) and the voltage synchronous controller (VSC), that generates the voltage wave comparing it to the one of the electric system to realize the exchange of reactive power. The control system of the STATCOM adjusts at each moment the inverse voltage so that the current injected in the network is in cuadrature to the network voltage, in these conditions $\mathrm{P}=0$ and $\mathrm{Q}=0$. 
In its most general way, the STATCOM can be modelled as a regulated voltage source $\mathrm{Vi}$ connected to a voltage bar Vs through a transformer, as the figure below shows. Fig. 3.

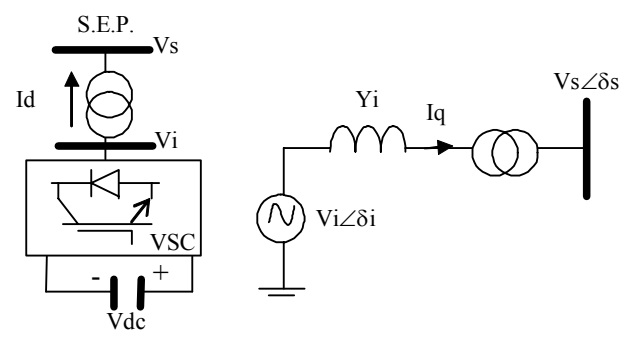

Fig. 3. STATCOM

Using the classical equations that describe the active and reactive power flow in a line in terms of $\mathrm{Vs}$ and $\mathrm{Vi}$, the transformer impedance (which can be assumed as ideal) and the angle difference between both bars, we can define $\mathrm{P}$ and $\mathrm{Q}$.

Where $\delta$ is the angle between $\mathrm{Vs}$ and $\mathrm{Vi}$ in this system. When the STATCOM operates with $\delta=0$ we can see how the active power send to the system device becomes zero while the reactive power will mainly depend on the voltage module. This operating condition means that the current that goes through the transformer (Iq) must have a $\pm 90^{\circ}$ phase difference to Vs. In other words, if $|\mathrm{Vi}|$ is bigger than $|\mathrm{Vs}|$, the reactives will be send to the STATCOM of the system (capacitive operation), originating a current flow in this direction. In the contrary case, the reactives will be absorbed from the system through the STATCOM (inductive operation) and the current will flow in the opposite direction. Finally if the modules of Vs and Vi are equal, there won't be nor current flow nor reactive flow in the system.

Thus, we can say that in a stationary state Q only depends on the module difference between $|\mathrm{Vs}|$ and $|\mathrm{Vi}|$ voltages.

There can be a little active power exchange between the STATCOM and the SEP. The exchange between active power between the inverter and the AC system can be controlled adjusting the output voltage angle from the inverter to the voltage angle of the AC system. This means that the inverter can not provide active power to the AC systems from the DC accumulated energy if the output voltage of the inverter goes before the voltage of the AC system. On the other hand, the inverter ca absorb the active power of the AC system if its voltage is delayed in respect to the AC systems voltage.

Figure 4. shows a simplified configuration of a STATCOM with a source of energy coupled to the DC side. The interface provides the coupling between the DC side of the STATCOM and other energy sources that can be of any kind of energy accumulation device or DC source: battery banks, DC generators, photovoltaic systems, or other power electronics device, where $\mathrm{P}_{\mathrm{mCD}}$ represents the active power of the DC source.

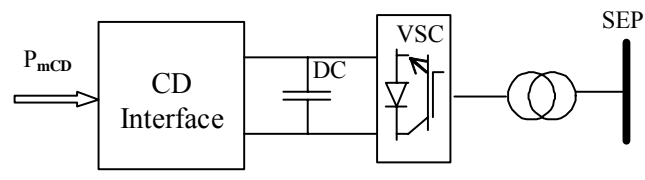

Fig.4. STATCOM with a source of energy

When this FACTS device is applied in distribution systems is called D-STATCOM (DistributionSTATCOM) and its topology is the same one with small modifications and adaptations, oriented to a possible future amplification of its possibilities in the distribution network at low and medium voltages, implementing the function so that we described as flicker dampfing, harmonic filtering and hole and short interruption compensation.

\section{B. SSSC (Static Synchronous Series Compensator)}

This device works the same was as the STATCOM. It has a voltage source converter serially connected to a transmission line through a transformer. It is necessaryy an energy source to provide a continuous voltage through a condenser and to compensate the losses of the VSC. A SSSC is able to exchange active and reactive power with the transmission system. But if our only aim is to balance the reactive power, the energy source could be quite small. The injected voltage can be controlled in phase and magnitude if we have an energy source that is big enough for the purpose.

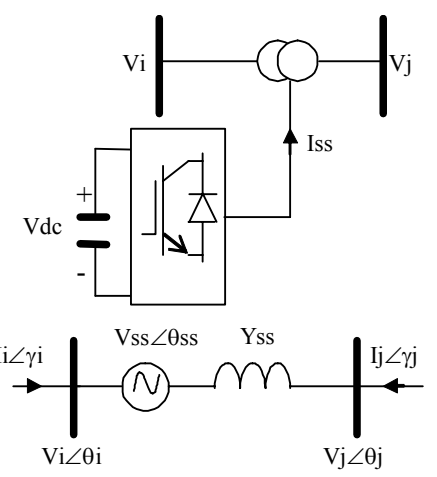

Fig. 5. SSSC

With reactive power compensation only the voltage is controllable, because the voltage vector forms $90^{\circ}$ degrees with the line intensity. In this case the serial injected voltage can delay or advanced the line current. This means that the SSSC can be uniformly controlled in any value, in the VSC working slot.

Thus, a SSSC can work like a controllable serial condenser and a serial reactance. The main difference is that the voltage injected through a SSSC is not related to the line intensity and can be controlled independently. This important feature means that the SSSC can be used with excellent results with low loads as well as with high loads.

We can also approve of the general use of a serial controllable condenser in the case of SSSC it controls the dynamic energy flow and improves the stability 
between the angle and the voltage. The fact that a SSSC can induce capacitive and inductive voltage increases the operation field of the device. During the stability slot the potential of dampfing electromechanical oscillations increases.

The transformer included in the scheme gives way to a cost disadvantage comparing to the controllable serial condensers. The transformer also reduces the efficiency of the SSSC. Due to its additional reactance. We will be able to solve this problem in the future by using SSSC's without transformer. The device also requires protection equipment that derivates the SSSC in case the line suffers of very high fault currents.

The SSSC version applied in distribution systems is the Dynamic Voltage Regulator (DVR) that basically consists on a three-phase converter connected to the distribution line through a serial connected transformer and with a load element (or source) in the DC side of the converter. A voltage to synchronize it with the line voltage can be injected (or in cuadrature with the current) of a variable amplitude and phase, and this allows the active and reactive power exchange between the line and the energy storage device, compensates holes in the network voltage and improves the unbalances between phases.

The Unified Voltage Controller UVC, is a cheap version of the DVR because it doesn't have the energy storage device, it is made to operate in network points with a high level of short circuit.

These devices correct the voltage when there is a fault in the network but also have a lot of advantages in normal use, when there are no disturbances, like:

- Due to the continuous voltage injection and in combination with a properly structured controller, it is possible to control the power factor of connected loads.

- In the interconnected distribution network topologies, the additional voltage with its controllable magnitude and phase, can be used to work on the power flows.

- It can also help to cover the capacitive reactive power demand in cable networks, which is higher than in aerial lines, mainly during low load periods that cause inadmissible load elevations.

- It balances loads in interconnected distribution networks, providing a balanced system.

- It reduces the harmonics caused because of the use of distributed electrical generation pants at a distribution network level, by active filtering by injecting voltage with the converter at the load side.

C. UPFC (Unified Power Flow Controller)
The most complete FACTS device is the unified energy flow controller. It is the only device with serial and parallel compensation operated by a common link of direct current. The serial compensator has a three-phase inverter and voltage source (2) that gives a serial-to-theline voltage through the winding of a serial transformer. The derivation compensator (basically a STATCOM) has an inverter(1) connected to a point of the line through a shunt transformer.

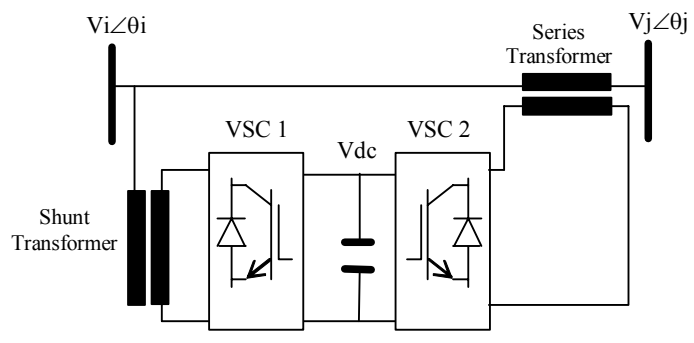

Fig. 6. UPFC

The 2 converter has the main function of the UPFC; it injects an $\mathrm{AC}$ voltage to the transmission line, which magnitude and angle are controllable through a serial transformer. The basic function of the 1 converter is to give or absorb the real power that the 2 converter demands in the common DC link. The 2 converter supplies or absorbs locally the required reactive power and exchanges the active power as a result of the serial injected voltage which varies in module and angle, as we can see in figure 7.

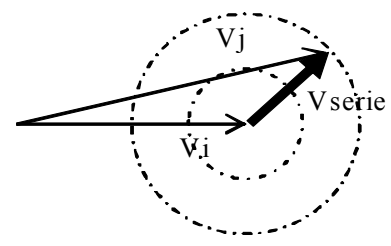

Fig. 7. Vj variation through Vserie

A UPFC system can regulate the active and reactive power at the same time. Generally it has three control variables and can operate in different modes. The converter connected in derivation regulates the distribution bar voltage and the serial connected converter regulates the active, reactive power and the voltage of the serial connected point. An UPFC can act and fulfil the functions that the other FACTS do, voltage support, energy flow control and improvement of the stability. Nevertheless the complexity and above all the cost of it made its installation possible only for certain cases.

\section{Conclusions}

Due to the, every time higher requirements of the liability and quality of the electricity, the implantation of devices capable of guaranteeing these requirements will keep increasing. The power electronic systems with a Voltage Controlled Source (VSC) topology connected to the network, have the ideal features to improve the capacity of the electric energy transmission of the networks. 
Even though this kind of systems have been applied in transport systems of high and medium voltages, the actual challenge it to implement these very same topologies in the electrical energy distribution networks. This is of important interest in lines that make a strong use of distributed generation, in which the quality of the transmission could be maimed by the connection of generation systems with low or null performances to ensure the energy supply under certain circumstances.

The development of high power inverters (more than 100MVA) of high performances at low cost is necessary to consolidate compensators such as STATCOM (Static Synchronous Compensator), SSSC (Static Synchronous Series Compensator) and UPFC (Unified Power Flow Controller). The developments spoil the potentialities of the multi-level converters to implement FACTS devices using the newest power semiconductor device technology. The multi-level converters are especially suitable to work at high voltages and low switching frequencies. The areas to improve would be these three: the converter topology, the basic control strategies and the applications of multi-level FACTS devices.

\section{References}

[1] Narain G. Hingorani and Laszlo Gyugyi. "Understanding FACTS. Concepts and Technology of Flexible AC Transmission Systems". IEEE Press, (1999)

[2] Vivas, J. Suárez, J. Expósito J. Simulación en ATP del compensador estático de potencia reactiva (statcom) utilizando técnicas de modulación SPWM. CIVIE 2004

[3] M. Aredes, G. Santos Jr., "A Robust Control for Multipulse STATCOMs", Proceedings of IPEC 2000, Vol. 4, pp. 21632168, Tokyo, 2000.

[4] Y.H. Song, A.T. Johns, Flexible ac transmission systems (FACTS). IEE Power and Energy Series 30, 1999.

[5] H. Fujita, S. Tominaga, H. Akagi, "Analysis and Design of a DC Voltage- Controlled Static Var Compensator Using QuadSeries Voltage Source Inverters," IEEE Trans. on Industry Applications, Vol. 32, No. 4, 1996

[6] L. Gyugyi, A. Ekstrom, N. Christl, "Conversores Controlados por Tensão e Capacitores Série CCAT", Curso e Workshop, Cigré Brasil, CE - 14, Furnas, Rio de Janeiro, 2000.

[7] Davalos, R. Ramirez, J. Caracteristicas funcionales del STATCOM. Cigre Mexico, Bienal 2001 